\title{
TEST DE AUTONOMÍA PSICOLÓGICA (TAP). UNA PRUEBA EN CONSTRUCCIÓN
}

\section{PSYCHOLOGICAL AUTONOMY TEST. A TOOL IN CONSTRUCTION}

\author{
Albert Vidal Raventós \\ Centro Simbòlics Psicoteràpia. Barcelona, España \\ Alejandro Garcia-Gutierrez \\ Universitat de Barcelona, España \\ Víctor Rouco \\ Ghent University, Bélgica
}

Cómo referenciar este artículo/How to reference this article:

Vidal Raventós, A., Garcia-Gutierrez, A. y Rouco, V. (2019). Test de Autonomía Psicológica (TAP). Una prueba en construcción. Revista de Psicoterapia, 30(113), 141-152. https://doi.org/10.33898/ rdp.v30i113.307

\begin{abstract}
Resumen
El Test de Autonomía Psicológica (TAP) es una prueba psicológica en desarrollo. Su objetivo es medir el sistema de regulación moral de un individuo, estableciendo un perfil de autonomía psicológica acorde con el Modelo del Desarrollo Moral (Villegas, 2011, 2013, 2015). En el presente artículo, los autores presentan una herramienta de evaluación de libre acceso online (http://www.autonomiapsicologica.com) Se aportan evidencias sobre la validez estructural del modelo a partir de una recolección de datos con 2032 sujetos.

Palabras clave: modelo del Desarrollo Moral, autonomía psicológica, test psicológico, test online, investigación de resultados en psicoterapia
\end{abstract}

\begin{abstract}
The Psychological Autonomy Test (PAT) is a psychological test in development. Its objective is to measure the moral regulation system of an individual, setting a psychological autonomy profile according to the Moral Development Model (Villegas, 2011, 2013, 2015). In this work the authors present an assessment tool freely available online (http:// www.autonomiapsicologica.com). Promising evidence of structural validity was found after collecting data on 2032 participants.

Keywords: Moral Development model, psychological autonomy, psychological test, online test, psychotherapy outcome research
\end{abstract}




\section{Introducción}

El concepto de Autonomía, desde el punto de vista psicológico, es la capacidad de regularse por uno mismo, de tomar decisiones y actuar en base a una norma o criterio propio. El término proviene del griego auto, "uno mismo", y nomos, "norma". Significa esencialmente capacidad de autodeterminación, de decidir según una voluntad intencional: en base a la elección de objetivos prefigurados de los que uno es responsable. Decisiones que son (o pueden ser) autónomas en la medida que se dan en un marco de libertad, aunque estén condicionadas inevitablemente por la facticidad histórica, social, familiar y material (Villegas, 2011).

La persona autónoma, pues, es aquella que toma decisiones libres y responsables en los diferentes ámbitos de su vida. Decisiones que siguen este proceso: intentan identificar lo que más conviene y satisface al sujeto, a la vez que adaptarse a las condiciones externas, tanto impersonales como interpersonales (Villegas, 2015). Decisiones que son a menudo complejas y contradictorias, pues enfrentan intereses en conflicto, y dan respuestas dolorosas por implicar alguna renuncia (Villegas, 2018). Autonomía es voluntad y lucha, aunque también es aceptación y renuncia. Es el arte de gestionar las diferentes tendencias en conflicto, y de sintetizar una buena respuesta para cada reto, que satisfaga la propia voluntad de forma negociada con la realidad del mundo.

Decisiones de diferente índole, que son referidas a las necesidades, a los deseos, a las normas sociales impersonales, o a las relaciones con los otros y las normas interpersonales que van asociadas. Se trata de diferentes ámbitos decisorios con diferentes implicaciones morales, que el Modelo del Desarrollo Moral(Villegas, 2011) inscribe respectivamente en los términos de prenomía (necesidades), anomía (deseos), heteronomía (norma impersonal) y socionomía (norma interpersonal). Cuatro ámbitos de regulación que evolutivamente siguen un proceso progresivo de aparición y desarrollo, y que en la fase adulta representan los cuatro subsistemas básicos de regulación moral. Autonomía y desarrollo moral, así, quedan estrechamente vinculados, en la medida que la primera es el reflejo de la óptima integración de los diferentes subsistemas de regulación moral.

El concepto de Desarrollo Moral, sin embargo, no es unívoco. Fue planteado inicialmente por Jean Piaget (1932/1976), como extensión de su propio trabajo sobre el desarrollo cognitivo en la infancia y la adolescencia. Según Piaget, a lo largo del crecimiento, el niño y la niña van desarrollando capacidad de juicio moral, en concordancia con sus logros cognitivos. Posteriormente, y partiendo de ese enfoque cognitivo-evolutivo, su discípulo Lawrence Kohlberg desarrolló un extenso trabajo sobre Desarrollo Moral, incluido un test, el Cuestionario de Razonamiento Moral (Colby, 1987). Dicho cuestionario se basa en el planteamiento de una serie de historias dilemáticas, tales como el dilema de Heinz y la medicina, donde el sujeto que es evaluado debe tomar una decisión frente al conflicto planteado. La prueba pone énfasis no tanto en la decisión tomada, como en las razones que llevan al entrevistado a tomar la decisión concreta. Se consideran aspectos como los intereses 
personales y las normas abstractas tenidas en consideración (la ley, la justicia). Finalmente, el entrevistador analiza las respuestas para determinar el nivel de desarrollo del juicio moral del entrevistado, que puede ser pre-convencional, convencional o post-convencional. La prueba proporciona, de ese modo, un indicador del nivel de desarrollo moral que la persona ha alcanzado, en una escala jerárquica de seis estadios consecutivos.

Posteriormente, y a partir del trabajo de Kohlberg, se han creado otros instrumentos psicométricos basados en su teoría, donde destacan el Moral Judgment Test (MJT) de George Lindt (2005) y el Defining Issues Test (DIT) de la Universidad de Minnesota (Rest, 1979). Estas nuevas propuestas ponen énfasis en elementos complementarios como la motivación y los afectos.

Sin embargo, y a pesar de su aparente similitud, el concepto de desarrollo moral en ambos modelos (Villegas y Piaget/Kohlberg) es significativamente diferente. Al margen de la diferencia en la concepción de la construcción genética y evolutiva de las etapas del desarrollo moral entre la concepción de Kohlberg y la de Villegas, puesta de manifiesto, por ejemplo, en la diversidad de criterios de clasificación para cada una de ellas o sobre el momento evolutivo de la aparición específica de alguna de ellas como la heteronomía, existen otras diferencias fundamentales que afectan a cuestiones de concepto y de aplicación.

La escuela neopiagetiana, con Lawrence Kohlberg (1964) a la cabeza, define la competencia del juicio moral como "la capacidad de tomar decisiones y emitir juicios morales (basados en principios internos) y de actuar en función de tales juicios". En esta definición el énfasis se pone en la formación del juicio moral, lo que implica una actividad básicamente criteriológica (razonamiento moral), que lleva, según el estadio evolutivo (pre-), (post-), -convencional del sujeto a poder juzgar sobre la mayor o menor (a)moralidad de una acción. Los test propuestos por estos autores plantean evaluar la rectitud de los criterios empleados para tomar sus decisiones por parte de los sujetos que se hallan en situaciones de conflicto entre principios morales, por ejemplo: robar una medicina en caso de necesidad (Kohlberg) o realizar espionaje por parte de los trabajadores de una empresa para demostrar prácticas abusivas por parte de sus directivos (Lind, 2000).

La propuesta de Villegas $(2011,2015)$ no se interesa tanto por la rectitud del juicio moral, que no deja de ser (pre-, post-), -convencional, sino por las implicaciones que tienen los distintos niveles de desarrollo moral en la regulación de la vida psíquica. Así la trascendencia del sistema de regulación pre-, a-, hetero- o socionómico no estriba en la capacidad de emitir juicios o de evaluar su adecuación moral, sino en las repercusiones que, para el bienestar psicológico consecuente, por ejemplo sentimiento de culpabilidad, vergüenza, rumiaciones, ansiedad, compulsiones, anhedonia, somatizaciones, etc., puedan tener los comportamientos derivados del predominio de un sistema de regulación moral sobre otro, o del conflicto surgido entre ellos.

Viktor Frankl, por ejemplo, amenazado como judío de ser deportado a un 
campo de concentración en época de la anexión de Austria a la Alemania nazi, tuvo que decidir entre quedarse con sus padres en Viena o aprovechar un visado personal que le había sido conseguido por la embajada suiza para trasladarse a EE.UU, donde hubiera quedado definitivamente a salvo (Frankl, 2016). El interés por conocer el criterio de regulación moral seguido por el fundador de la Logoterapia no se centraría tanto en evaluar el grado de moralidad de su decisión, sino en comprender las motivaciones que le empujaran a tomarla y las repercusiones psicológicas que pudieran derivarse de ella.

En este contexto era posible tomar decisiones, como la de utilizar el visado para refugiarse en EE.UU. que, según el modelo de Kohlberg, corresponderían a un criterio preconvencional. Era posible igualmente decidir quedarse con sus padres, siguiendo los preceptos de la ley mosaica de "honrar padre y madre", afrontando el riesgo de ser deportado a un campo de concentración, como así sucedió finalmente, que se regiría por un criterio convencional.

La visión del Modelo del Desarrollo Moral de Villegas no juzga el grado de moralidad de la decisión tomada por Frankl, sino la posible integración o desintegración de los diferentes sistemas de regulación implicados en la decisión y las consecuencias sobre su equilibrio psicológico: por ejemplo, los sentimientos de culpa en el caso de ponerse a salvo individualmente, o de rabia, miedo o tristeza en el caso de deportación. Tales sentimientos solo serían asumibles desde una decisión autónoma.

Otra diferencia importante es la perspectiva teleológica o finalista que preside el planteamiento psicoterapéutico de Villegas (2011, 2013, 2015), orientado a la consecución de la autonomía, como máxima expresión de la libertad humana. Para el autor la constricción de la libertad se encuentra en la base de cualquier patología neurótica en la medida en que son los dilemas morales los que ponen en juego su ejercicio espontáneo, dando origen a los trastornos ansioso-depresivos. Su interés se centra en la comprensión de la psico(pato)logía desde esta perspectiva y en la posibilidad de una psicoterapia orientada al desarrollo de la autonomía, como punto de integración de los diversos sistemas de regulación moral.

Como consecuencia, el ámbito de aplicación de los modelos neopiagetianos es preferentemente el de la pedagogía, a saber, la formación de ciudadanos éticamente conscientes y responsables, tal como ponen de relieve los propios tests dedicados a explorar los diversos niveles de desarrollo moral, donde se plantean cuestiones de comportamiento social o público. El ámbito de aplicación del modelo de la Terapia del Desarrollo Moral, en cambio, se centra en favorecer "el proceso de convertirse en persona autónoma" (Villegas 2015), dando prioridad a la dimensión individual, aunque sin excluir la dimensión social o cívica, inherente a la propia responsabilidad de un sujeto autónomo. Así, en la medida que autonomía, regulación moral y psicopatología se conciben como conceptos interconectados, el ámbito de aplicación de dicho modelo es preferentemente clínico. Así como el test que presentamos en el presente artículo. 


\section{El Test de Autonomía Psicológica (TAP)}

Se trata de una herramienta psicométrica pensada para el ámbito clínico, y creemos que especialmente adecuada tanto para comprender la organización del sistema de regulación moral en el momento de iniciar la terapia, como para monitorizar los cambios producidos a través del proceso terapéutico, como feedback tanto para el terapeuta como para el paciente para conocer el progreso en autonomía psicológica a lo largo de la terapia.

La monitorización del proceso terapéutico es actualmente un objetivo prioritario en la investigación sobre psicoterapias basadas en la evidencia. El uso de instrumentos adecuados ayuda a obtener una medida más exhaustiva del progreso y su contraste entre enfoques, centros y profesionales. Comúnmente se ha utilizado observación directa, entrevistas, autorregistros (Feixas et al., 2018). Técnicas que pueden resultar excesivamente cualitativas o bien costosas, por lo que es interesante el uso de herramientas de registro de tipo cuantitativo y de aplicación ágil. La mayoría de los instrumentos que existen actualmente se centran en medir la sintomatología de cada patología en particular, siendo escasamente aplicables a pacientes con otras problemáticas o con diagnósticos inciertos o poco definidos (Twigg et al., 2009).

Por ese motivo, existe la necesidad de elaborar herramientas breves que eviten el uso de múltiples instrumentos y recursos. También que fomenten una evaluación cuantitativa del proceso terapéutico de modo que facilite la integración y análisis de la información proveniente de diferentes profesionales, ámbitos y patologías (Feixas et al., 2018).

Una evaluación del cambio durante la terapia que resulta especialmente interesante no sólo para el estudio de la eficacia, sino también como retroalimentación para el terapeuta y el propio cliente (Feixas et al., 2012). En nuestro caso, proporcionando un perfil de regulación que indicaría el progreso en el desarrollo de la autonomía, y orientaría en la detección de los déficits de regulación que aún podrían persistir, para adaptar así el trabajo terapéutico.

Para Villegas $(2011,2015)$, la regulación autónoma es la capacidad de decidir en el ámbito personal, relacional y social, y requiere del pleno desarrollo de los subsistemas de regulación moral. La ausencia de autonomía, por el contrario, indica déficits de regulación, que el test mediría en función de la mayor o menor presencia de los diferentes subsistemas de regulación. El concepto de autonomía es, así, central en el modelo, motivo por el cuál ha sido escogido para poner el nombre de la nueva herramienta: Test de Autonomía Psicológica.

La función de la autonomía, pues, es conseguir la integración de todos los subsistemas de regulación moral (Villegas, 2018). Se trata de una tarea permanente, laboriosa y constante, siempre en tensión dialéctica en búsqueda de una buena integración. Sin embargo, cabe distinguir aquí integración de autonomía, pues no son sinónimos. Se considera integración la ausencia de discrepancia entre los diferentes subsistemas de regulación, y por lo tanto refleja ausencia de conflicto 
psicológico (Feixas, 2011). Lo que no presupone necesariamente autonomía psicológica. Por ejemplo, un niño puede actuar de forma integrada en función de su estadio evolutivo, sin por ello ser autónomo. Del mismo modo, una persona adulta puede presentar un desequilibrio de regulación, donde la anomía (deseo) ocupa un lugar preeminente y, a pesar de ello, puede no tener dificultades ni discrepancias con su entorno, si éste se ha adaptado a sus demandas. En este caso, diríamos que esta persona se regula de forma integrada -ausencia de conflicto psicológico (egosintónica)-, aunque lejos de ser autónoma - por el desequilibrio de regulación. De este modo, cabe señalar que el objetivo de la herramienta psicométrica no está orientado a la detección del grado de integración, sino a describir el perfil de regulación moral y el grado de autonomía asociado.

Con anterioridad, se publicó un cuestionario de regulación moral (Villegas, 2015), con carácter exploratorio y divulgativo, elaborado por Solsona, Alabart, Oliveras, Creus, Gómez Enguix y Vidal Raventós. Se basaba en la medición del peso relativo de cada subsistema de regulación, como es nuestro caso. El actual trabajo recoge aquella experiencia, aunque con herramientas y objetivos diferentes.

\section{Descripción del test}

El test se encuentra online (http://www.autonomiapsicologica.com), bajo el título "Test de Autonomía Psicológica". Su dirección está indexada por Google y puede aparecer en los resultados de búsqueda utilizando determinadas palabras clave. No se ha promocionado con publicidad ni se ha enlazado en ninguna página web. Es un test de libre acceso y gratuito, cualquier persona puede acceder al mismo utilizando internet. Las repuestas de los sujetos se van almacenando en una base de datos a medida que van avanzando.

Al abrir la primera página del link referenciado aparece en pantalla este tarjetón

\section{¿Eres una persona autónoma?*}

\section{*Este test se encuentra aún en fase experimental.}

\section{Te damos la bienvenida al Test de Autonomía Psicológica. Te llevará tan solo entre 5 - 10 minutos y es totalmente gratis. Al final podrás conocer tus resultados.}

En este estudio se ha buscado crear un test que permita describir el perfil de regulación moral de cada persona a partir del peso relativo de cada subsistema de regulación. Cómo la persona se regula en el día a día, y cuál es su flexibilidad para priorizar un subsistema de regulación u otro en función de su voluntad o la conveniencia de cada situación, reflejando de este modo su grado de autonomía.

Con este objetivo en mente, a partir de los datos recabados en este estudio se intenta estimar cinco factores latentes que se supone subyacen al TAP. Cada uno de estos se corresponde con un sistema de regulación moral. El modelo de cinco factores correlacionados se ajusta a los datos. El test está formado por varias 
situaciones con cinco ítems asociados. Se plantea la hipótesis, por tanto, que, dentro de cada situación, estos ítems compartirán una variabilidad específica.

Todo esto debería permitir la validación el test, aportando datos cuantitativos que avalen algunos de los postulados de la Teoría del Desarrollo Moral, aunque serán necesarios estudios adicionales que apoyen con más evidencia la utilidad de este modelo teórico.

\section{Método}

\section{Participantes}

Los participantes en esta fase experimental de la construcción del test, han sido voluntarios anónimos que han respondido a la invitación de rellenar el test a través de un acceso libre en internet. De este modo, aunque la muestra inicial alcanzó los 2860 sujetos, se eliminaron todos aquellos que no habían terminado el test, quedando finalmente en 2032 sujetos, que completaron con éxito el test online. La muestra total se ha ido recogiendo durante el periodo de un año aproximadamente (desde que se hizo público en la red hasta el momento del análisis de datos). Se parte pues de un muestreo aleatorio, para poder valorar la consistencia de los ítems, sin pretensiones todavía de valor predictivo, en función de criterios diagnósticos o clínicos

\section{Instrumento y medidas}

En la primera página del test, como se puede ver más arriba, se explica que el test durará aproximadamente entre 5 y 10 minutos y que es totalmente gratuito. Posteriormente, cuando el sujeto da al botón de continuar, se pregunta a los sujetos si ya lo han realizado con anterioridad para evitar tener medidas repetidas. En caso afirmativo, los datos no se guardan.

En la página siguiente se recaban varios datos. La edad y el género son obligatorios, se pide que los sujetos tengan como mínimo 16 años. A parte de esto también se pide de forma voluntaria datos sobre: satisfacción vital (escala de 1 a 10), estado civil, últimos estudios, ocupación, país de nacimiento, ciudad de nacimiento, país de residencia, código postal actual.

Después de introducir estos datos se presentan brevemente las instrucciones del test, describiendo la actitud que se debe mantener durante el mismo y avisando de que no se podrá utilizar el botón de retroceso. Cuando el sujeto presiona el botón, el test da comienzo.

El test está formado por 12 situaciones. Cada una de ellas tiene asociados 5 ítems, que se corresponden con los diferentes sistemas de regulación moral. Cada ítem representa una forma de responder a la situación en función de ese sistema. El test ha sido construido de este modo para aportar un marco de referencia al sujeto y así disminuir la variabilidad intra-individual (Lievens, De Corte, Schollaert, 2008). 
A continuación puede verse un ejemplo de situación con sus respectivos ítems:

Situación: Inesperadamente me comunican que hoy tengo el día libre. ¿Qué hago?

Ítem 1 (Prenomía): Voy dejando pasar el día sin hacer nada en concreto. Ítem 2 (Anomía): Rápidamente encuentro un montón de cosas apasionantes que me gustaría hacer.

Ítem 3 (Heteronomía): Lo primero que hago es organizar el día, elaboro un plan.

Ítem 4 (Socionomía complaciente): Pienso en mis amigos y a quién podría llamar para quedar.

Ítem 5 (Socionomía vinculante): Aprovecho para estar con los míos, siempre hay cosas que puedo hacer por ellos.

Las situaciones y los ítems se presentan de manera aleatoria para cada sujeto utilizando un algoritmo. Las situaciones son presentadas primeramente con un grafismo aumentado, cuando el sujeto da al botón de continuar, aparece el primer ítem asociado a esta situación junto con una escala visual analógica y, de nuevo, la situación con el grafismo disminuido. Todos los ítems son presentados de la misma manera hasta llegar a la siguiente situación.

El test está diseñado para que se adapte a los diferentes dispositivos que se utilizan hoy en día para acceder a internet: móviles, tablets, ordenadores, etc. Por esta razón, la escala visual analógica que el sujeto utilizará para responder también se adapta al tamaño de la pantalla, esto quiere decir que a veces será más corta y otra más larga. La escala es una barra con un botón deslizante, un diseño al que todos estamos acostumbrados a utilizar hoy en día. Al mover el botón, un texto indica la puntuación que se ha seleccionado, esta oscila entre 0 y 100, que se encuentra en los extremos. Debajo de ellos, una leyenda reza: " $0=$ no me representa en absoluto" y " $100=$ me representa totalmente". De cada ítem se almacena la puntuación y también el tiempo que una persona ha tardado en responderlo.

Cuando el sujeto ha contestado a todas las situaciones, el test finaliza, se presentan unos resultados tentativos basados en las respuestas del sujeto (se advierte al sujeto de tomarlos con precaución) y se explica resumidamente el significado de los diferentes sistemas de regulación. También se da la oportunidad de que el sujeto comparta los resultados en las redes sociales y de este modo, incentivar la difusión del test. Los resultados del test se presentan gráficamente. Encontramos en Mallor y Villegas (2011) una primera propuesta de representación geométrica de los diferentes subsistemas de regulación: en forma de círculos de distinto tamaño, cada uno de ellos correspondiente a uno de los subsistemas. En este caso se ha utilizado el gráfico de radar con cinco ejes de diferente dimensión, en función del peso relativo de cada subsistema de regulación moral. El resultado gráfico es un pentágono de tamaño y regularidad variable según el equilibrio entre sistemas de regulación (ver, por ejemplo, figura 1). 


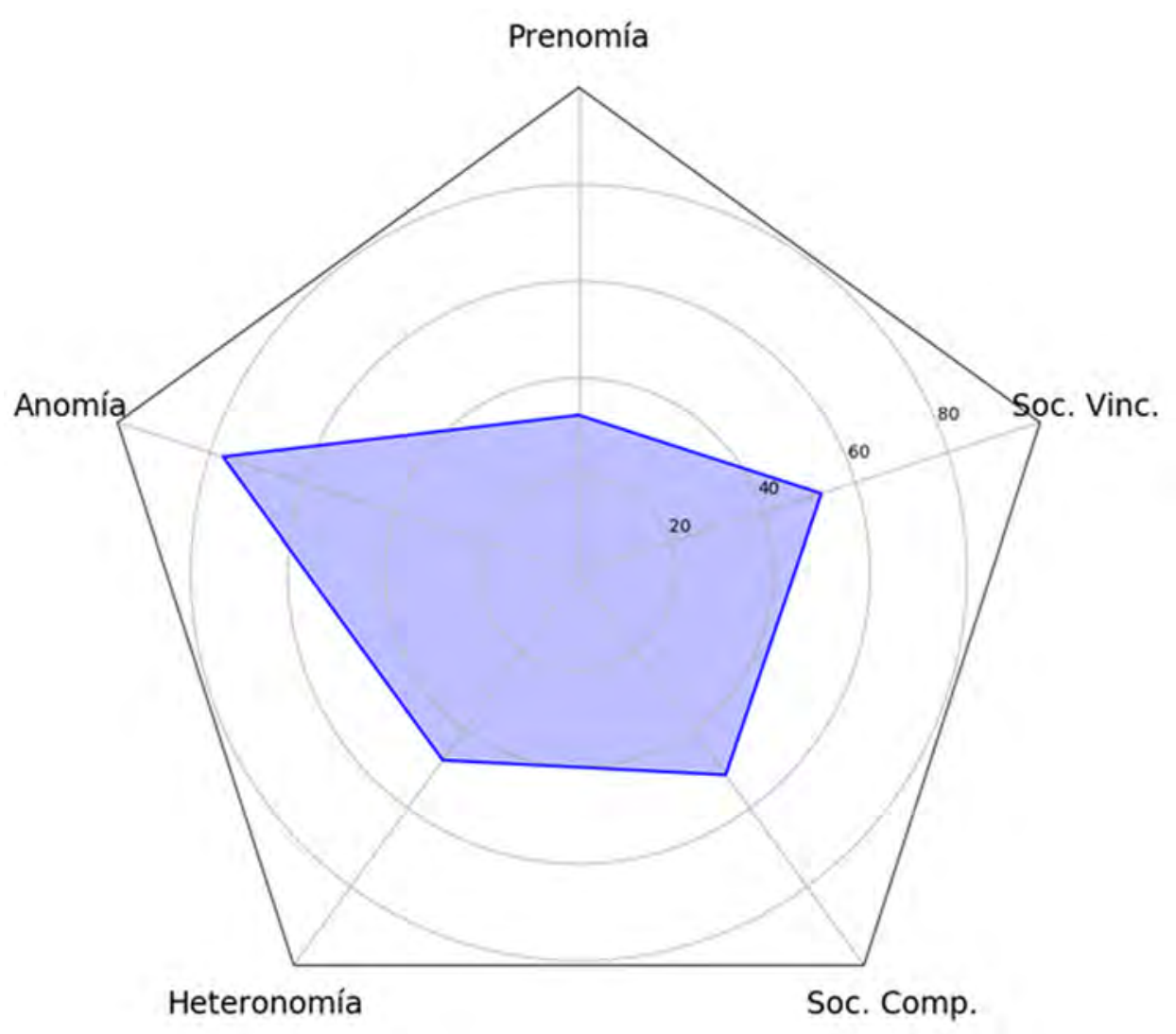

Figura 1. Gráfico de radar.

\section{Procedimiento}

La programación del test se llevó a cabo utilizando el lenguaje de programación Python 3, y para el diseño, en el que se priorizó la sencillez y facilidad de uso, se utilizó CSS y JavaScript. Los análisis estadísticos se llevaron a cabo utilizando R.

Las situaciones y los ítems del test fueron elaborados cuidadosamente, pasando varios procesos de cribado y corrección, teniendo en cuenta que fuesen suficientemente comunes y realistas para que todo el mundo pudiese sentirse representado al leerlas.

\section{Análisis de datos}

El estilo de respuesta del test es de estímulo único, utilizando una escala analógica de respuesta. Este tipo de escalas tienen la peculiaridad de generar datos continuos, pero la complicación de generar un mayor número de respuestas en los límites del rango (efectos suelo y techo). Observamos habituales formas en "u"” en los histogramas de frecuencia de los ítems. Por este motivo hemos hecho una 
transformación logarítmica a los ítems, que soluciona problemas relacionados con la violación de los supuestos de Máxima Verosimilitud.

Las respuestas transformadas se analizarán en un Análisis Factorial Confirmatorio (AFC). Se asumirá una estructura de clústers independientes, medidas congenéricas y factores latentes correlacionados en un primer AFC. Los ítems han sido elaborados por expertos en el modelo, por lo que la estructura factorial a priori es conocida y no vemos necesario el uso de un Análisis Factorial Exploratorio previo. Tras el primer AFC se ajusta otro modelo con los errores correlacionados de los ítems que comparten un marco de referencia o situación. A partir de entonces se irán eliminando ítems en AFC sucesivos, basándonos en índices de modificación y la matriz de correlación de los errores. Cada nuevo modelo se comparará con el anterior a través de pruebas de Chi cuadrado.

\section{Resultados}

La base de datos completa, usando únicamente las filas sin datos perdidos, es de 2032 sujetos. Respuestas con latencia de respuesta muy alta (más de 30 segundos) o muy baja (menos de 2 segundos) fueron consideradas valores perdidos.

El modelo que mejor se ajusta a los datos cuenta con 25 items, medidos en base a seis marcos de referencia o situaciones diferentes. El modelo final tiene un ajuste relativo a los datos. Según los puntos de corte clásicos sugeridos por Hu y Bentler (1998), el modelo tiene un buen ajuste acorde al RMSEA (95\% I.C. $=.030-.035)$ y al SRMR (.05). No obstante, el ajuste medido con el CFI está ligeramente por debajo del punto de corte sugerido por los autores citados anteriormente (.92).

Algunos factores latentes están fuertemente correlacionados. Hemos comprobado estructuras factoriales alternativas, agrupando factores más próximos según la teoría, y no ha resultado mejor que la estructura de cinco dimensiones.

\section{Discusión}

En Test de Autonomía Psicológica (TAP) es una prueba psicométrica en construcción. En el presente artículo se describen los primeros resultados, que muestran validez de constructo del modelo utilizado.

Por sus características descritas, este test (Garcia-Gutierrez, Vidal Raventós y Rouco, 2018) podría ser de utilidad para aquellos clínicos que desean conocer el perfil de regulación moral de sus clientes, aunque aún es pronto para concluir si este test es capaz de, por ejemplo, discernir los cambios que se pueden producir durante un proceso psicoterapéutico. Creemos que podría ser una herramienta útil para confirmar o desmentir las hipótesis clínicas del terapeuta. Su corto tiempo de aplicación, su naturaleza online, y el hecho de que se pueda realizar desde cualquier dispositivo, puede permitir que el cliente lo complete sin descontar tiempo de la sesión terapéutica. También se podría desarrollar una presentación en papel, si hubiera una demanda al respecto, pero complicaría su proceso de evaluación, ahora automatizado. 
Sería necesario repetir este test, en su versión breve de 25 ítems ajustada a los datos, con una muestra más controlada, por ejemplo en contextos clínicos o educativos. Además, será necesario seguir investigando en esta línea, para saber si este test tiene validez predictiva. Para ello, se podría intentar someter a prueba alguna de las implicaciones teóricas derivadas del Desarrollo Moral, y comprobar, por ejemplo, si las personas con un determinado déficit en alguno de los sistemas de regulación moral presentan un trastorno psicológico tipificado en el Manual Diagnóstico y Estadístico de los Trastornos mentales (5. ${ }^{\mathrm{a}} \mathrm{Ed}$.; DSM-V; American Psychiatric Association [APA], 2013). Posteriores investigaciones ayudarán a determinar la utilidad de este test y algunas de las conceptualizaciones del modelo teórico.

\section{Limitaciones del estudio}

Se han detectado algunas limitaciones de la prueba que deberían tenerse en cuenta en posteriores investigaciones.

Debido a que los participantes de este estudio son usuarios anónimos de internet, se ha ejercido un bajo control sobre la muestra. Pese a que se han descartado aquellos sujetos que contestaban por debajo de un umbral de tiempo (es decir, que probablemente contestaban a los ítems sin leeros) no se han utilizado mecanismos efectivos para evitar que los sujetos den respuestas falsas o incorrectas. Tampoco se puede estar totalmente seguro que un mismo sujeto no haya repetido el test varias veces o que hayan participado menores de edad que aún no hayan tenido la oportunidad para desarrollar los diferentes sistemas de regulación. Más importante es no poder estar totalmente seguros sobre el género de los participantes, ya que la muestra podría estar sesgada en una dirección. También es posible que los sujetos pertenezcan en su mayoría a un determinado país, cultura, estrato social, etc. que esté afectando a los resultados. A pesar de ello, la magnitud de la muestra permite suponer un grado aceptable de representatividad.

El hecho de que la escala se adapte al tamaño de la pantalla del dispositivo desde el cuál se está visualizando el test, provoca que la escala analógica cambie de resolución. Eso pudiera dar lugar a que algunos sujetos no hayan podido contestar con la precisión suficiente, por ejemplo, si lo completaban a través del teléfono móvil.

La naturaleza de este estudio tampoco ha permitido controlar el estado psicológico o la situación ambiental de los sujetos, cuya variación podría afectar los resultados de algún modo.

\section{Consideraciones finales}

El objetivo de la publicación del presente trabajo sobre el TAP, todavía en fase de construcción, es, entre otros, el de animar a terapeutas y estudiosos a involucrarse y colaborar en su desarrollo. Los datos recogidos de forma sistemática en contextos controlados han de permitir someter a validación su utilidad como instrumento de 
trabajo en distintos ámbitos de aplicación. Naturalmente queda mucho trabajo todavía por hacer y por eso animamos a quienes estén interesados en promoverlo a ponerse en contacto con nosotros.

\section{Referencias Bibliográficas}

American Psychiatric Association (2013). Manual Diagnóstico y Estadístico de los Trastornos Mentales (5. ${ }^{\mathrm{a}}$ Ed.). Madrid, España: Panamérica.

Colby, A., Kohlberg, L., Abrahami, A., Gibbs, J., Higgins, A., Kauffman, K., ... Tappan, M. (1987) The Measurement of Moral Judgment, Volume 1: Theoretical Foundations and Research Validation. Cambridge, MA: Cambridge University Press.

Feixas, G. (2011). Entrevista con Manuel Villegas sobre "El Error de Prometeo. Psico(pato)logía del Desarrollo Moral). Revista de Psicoterapia, 22(85), 111-123. Recuperado de: https://bit.ly/2LJ9ak3

Feixas, G., Evans, C., Trujillo, A., Saúl, L., Botella, L., Corbella, S., González, E., Bados, A., García-Grau, E. y López-González, M.A. (2012). La versión española del CORE-OM: Clinical Outcomes in Routine Evaluation. Revista de Psicoterapia, 23(89), 109-135. Recuperado de: https://bit.ly/31XTfnw

Feixas, G., Badia, E., Bados, A., Medina, J. C., Grau, A., Magallón, E., Botella, L. y Evans, C. (2018). Adaptación y propiedades psicométricas de la versión española del YP-CORE (Young Person's Clinical Outcomes in Routine Evaluation). Actas Esp Psiquiatr, 46(3),75-82.

Frankl, V. E. (2016). Lo que no está escrito en mis libros. Memorias. Barcelona, España: Herder.

Garcia-Gutierrez, A., Vidal Raventós, A., y Rouco, V. (2018). Autonomía psicológica (prueba y archivo de datos). Recuperado de: http://www.autonomiapsicologica.com

Hu, L. y Bentler, P. (1998). Fit Indices in Covariance Structure modeling: Sensitivity to underparameterized model misspecification. Psychological Methods, 3, 424-453.

Kohlberg, L. (1964). Development of moral character and moral ideology. En M. L. Hoffman y L. W. Hoffman, (Ed.), Review of child development research, Vol.I (pp. 383-431). Nueva York, NY: Russel Sage.

Kohlberg, L. (1984). The Psychology of Moral Development: The Nature and Validity of Moral Stages (Essays on Moral Development, Volume 2). San Francisco, CA: Harper \& Row.

Lievens, F., De Corte, W. y Schollaert, E. (2008). A closer look at the frame-of-reference effect in personality scale scores and validity.Journal of Applied Psychology, 93(2), 268-79. http://doi.org/10.1037/0021-9010.93.2.268

Lind, G. (2000). Moral regression in medical students and their learning environtment. Revista Brasileira de Educaçao Médica, 24(3), 24-33.

Lind, G. (2005). Validation and certification procedure for the Moral Judgment Test. Recuperado de: http:// www.uni-konstanz.de/ag-moral/mut/mjt-certification.htm

Mallor, P. y Villegas, M. (2011). Aplicación didáctica del modelo del desarrollo moral. Revista de Psicoterapia, 22(88), 55-79. Recuperado de: https://bit.ly/303Utth

Piaget, J. (1976). Le jugement moral chez l'enfant, París, Francia: PUF.

Rest, J. (1979). Development in judging moral issues. Minneapolis, MN: University of Minnesota Press.

Twigg, E., Barkham, M., Bewick, B., Mulhern, B., Connell, J. y Cooper, M. (2009). The Young Person's CORE: Development of a brief outcome measure for young people. Counselling and Psychotherapy Research, 9(3), 160-168, http://doi.org/10.1080/14733140902979722

Villegas, M. (2011). El error de Prometeo. Psico(pato)logía del desarrollo moral. Barcelona, España:Herder.

Villegas, M. (2013). Prometeo en el diván. Psicoterapia del desarrollo moral. Barcelona, España: Herder.

Villegas, M. (2015). El proceso de convertirse en persona autónoma. Barcelona, España: Herder.

Villegas, M. (2018). La teoría del desarrollo moral en el marco del análisis existencial. Revista de Psicoterapia, 29(109), 1-38. https://doi.org/10.33898/rdp.v29i109.226 